Author(s): H. W. Chen, C. J. Aine, E. R. Flynn, C.C. Wood

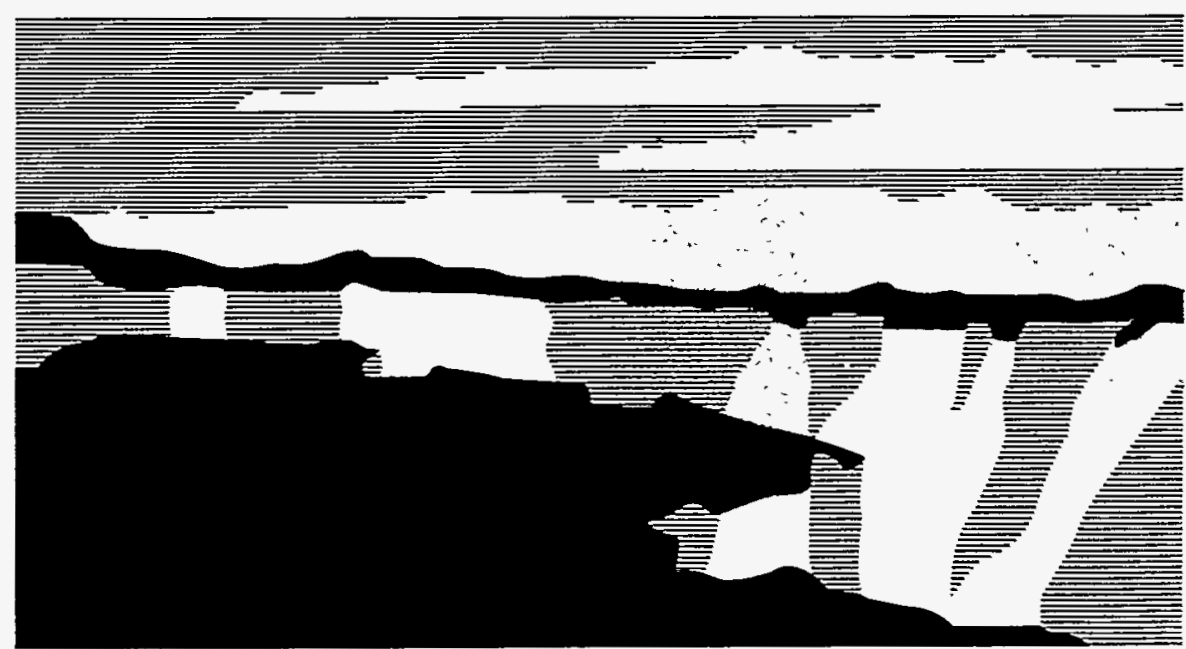

Los Alamos National Laboratory, an affirmative action/equal opportunity employer, is operated by the University of California for the U.S. Department of Energy under contract W-7405-ENG-36. By acceptance of this article, the publisher recognizes that the U.S. Government retains a nonexclusive, royalty-free license to publish or reproduce the published form of this contribution, or to allow others to do so, for U.S. Government purposes. The Los Alamos National Laboratory requests that the publisher identify this article as work pertormed under the auspices of the U.S. Department of Energy. 


\section{DISCLAIMER}

This report was prepared as an account of work sponsored by an agency of the United States Government. Neither the United States Government nor any agency thereof, nor any of their employees, makes any warranty, express or implied, or assumes any legal liability or responsibility for the accuracy, completeness, or usefulness of any information, apparatus, product, or process disclosed, or represents that its use would not infringe privately owned rights. Reference herein to any specific commercial product, process, or service by trade name, trademark, manufacturer, or otherwise does not necessarily constitute or imply its endorsement, recommendation, or favoring by the United States Government or any agency thereof. The views and opinions of authors expressed herein do not necessarily state or reflect those of the United States Government or any agency thereof. 


\title{
Nonlinear Spatio-temporal Interactions and Neural Connections in Human Vision Using Transient And M-Sequence Stimuli
}

\author{
Chen, H.-W., Aine, C.J., Flynn, E.R., and Wood, C.C. \\ Biophysics Group, MS D-454, Los Alamos National Laboratory \\ Los Alamos, NM 87545, U.S.A.
}

\section{INTRODUCTION}

There are two classes of connections among neurons in cat and monkey cortices [1]: (a) the traditional receptive field connections (feedforward connections), and (b) the dynamic association of neurons in different cortical areas which form functionally coherent assemblies (reciprocal connections). The feedforward connections have been well studied using traditional single-unit recording techniques. As pointed out by Singer [1], there are two strategies for characterizing the dynamic association of neurons in different cortical areas (reciprocal connections): 1) examining changes in discharge rate, and 2) examining temporal synchrony (the stimulus evoked coherent oscillations are in the gamma range $30-110 \mathrm{~Hz}$ ). The first was evidenced by spatio-temporal interactions beyond the classical receptive fields (RFs) for V1 cortical neurons. The second was revealed recently using multiple-unit recording techniques and cross-correlation analysis, which have shown that cortical neurons can synchronize their discharges very precisely (e.g., [2]). The reciprocal connections can be categorized as tangential intra-areal, interhemispheric, and/or feedback (between cortex and LGN) connections, and may play an important role in integrating and binding widely distributed and parallel representations in different cortical areas.

Reciprocal connections, in essence, are the dynamic wiring (connections) of the neural network circuitry [1]. Given the high complexity of the neural circuitry in human brain, it is quite a challenge to study the dynamic wiring of highly parallel and widely distributed neural networks. The measurements of stimulus evoked coherent oscillations provide indirect evidence of dynamic wiring. In this study, in addition to the coherent oscillation measurements, we provide two more techniques for testing possible dynamic wiring: 1) measurements of spatio-temporal interactions beyond the classical receptive fields, and 2) neural structural testing using nonlinear systems analysis.

Spatio-temporal interaction and its relation to reciprocal connections. Animal studies have revealed the existence of facilitatory and/or inhibitory integration fields (IF) beyond the classical receptive fields (RFs) for retinal ganglion cells and striate cortical neurons. The extent of the integration fields is about 2-5 times the classical receptive field size [3]. Polat and Sagi [4] have recently found, in human vision, suppression and facilitation lateral interactions between spatial frequency (SF) channels revealed by lateral masking psychophysical experiments conducted at the fovea. The mechanism mediating these interactions at different eccentricities is, however, still an open question. One hypothesis for the interactions is based on the existence of multiple spatial frequency (SF) channels in the human visual system. Each channel may involve many neurons, and the receptive field of an SF channel may be defined as overlapping receptive fields of all the involved neurons (i.e., the RF of an SF channel is larger than that of a single neuron). Therefore, the spatial lateral interaction beyond the classical RFs of single neurons may be explained by the interactions between neighboring SF channels (feedforward connections) having spatially overlapping receptive fields. Another hypothesis is that the multiple SF channels are parallel and distributed in different cortical areas, and they are connected and bind via dynamic wiring. In this case, the distance of spatial lateral interactions would be large. Indeed, Ts'o and coworkers [5] have found excitatory horizontal intracortical connections from cat primary visual cortex, and suggested that these connections might contribute to properties beyond classical $\mathrm{RF}$ analysis. Therefore, measurements of spatio-temporal interactions may provide information on both traditional feedforward and reciprocal connections.

Nonlinear Systems analysis and Neural Circuitry testing. Several approaches to studying unknown biological systems consider the system initially as a "black box." In one approach, knowledge of the input-output (I/O) behaviors of the system are acquired by identifying the system's I/O transfer function. For example, the Laplace transfer function of a linear system is directly related to the I/O relationship of the system described by a linear differential equation. Unlike analyses of simple linear systems, analyses for nonlinear systems present a greater challenge since higher-order transfer functions are required for full characterization of a nonlinear system's I/O behaviors. One method used to characterize a nonlinear system (which is generally time-invariant or stationary, causal, and continuous), expresses the $1 / O$ relationship in terms of Volterra or Wiener functional expansions [6]. Such expansions are fully specified by a set of Volterra (or Wiener) kernels which can be estimated from the I/O measurements of the system under study. The system kernels provide a characterization of the system I/O relationship, and thus allow one to predict the response of a physical or physiological system to an arbitrary stimulus. Furthermore, the measured lower-order (the 1st- and 2nd-order) system kernels can provide information about the internal structure of the practical system under study [7]-[9], which suggests a number of potential applications in the areas of neural system modeling and identification. For example, we are interested in characterizing the $I / O$ relationship of popula- 
tions of neurons in cortical areas of human brain, obtained from noninvasive electromagnetic measurements. Neuronal responses can be modeled as circuitry, and nonlinear analysis may ultimately allow one to characterize the structure of neural system circuitry, using system kemels.

We have recently developed techniques using sparse-stimulation and short m-sequence stimuli [10], and have successfully measured the 1st- and 2 nd-order kernels from neuromagnetic responses. The advantages for using this method are: 1) easier and fast kernel calculation; 2) short data acquisition times and less measurement errors; 3) short m-sequences with different lags (delays) are orthogonal to one another which permit their use in studying multiple input systems (i.e., the simultaneous presentation of $\mathrm{m}$-sequences). Furthermore, the results from MEG experiments [10] indicate that the $S / N$ ratio of responses evoked by m-sequences can be 2-3 times higher than responses evoked by transient target stimuli when stimulus presentation time is equated.

In this study, we have measured spatio-temporal interactions in human vision using transient and $\mathrm{m}$-sequence stimuli. The estimated cross-correlations (system kemels) obtained using m-sequence stimuli were examined in hope of identifying possible neural connections (structures) and mechanisms underlying both feedforward and reciprocal connections. Measurements of coherent oscillations using transient stimuli will also be briefly discussed.

\section{METHODS}

Evoked neuromagnetic fields were recorded with a BTi 7-channel SQUID-coupled gradiometer system in a magnetically-shielded chamber. Four subjects with normal vision (HWC, CA, WEN, and JE) participated in this study. In the transient studies, we presented two pattern onset target stimuli (circular sinusoidal gratings, $266 \mathrm{~ms}$ duration and $\sim 1 \mathrm{~Hz}$ rate of presentation) either simultaneously or sequentially with different SFs, to a specific eccentricity. These paired stimuli were presented at different eccentricities $\left(0.8^{\circ}, 1.6^{\circ}, 3^{\circ}\right.$, and $\left.6^{\circ}\right)$ in the visual field. The spatial separation between them was varied in order to quantify the nonlinear summation area. The nonlinear summation index was calculated as the peak response to the simultaneous presentation of stimuli divided by the sum of the peak responses to the two separate stimulus presentations. In the m-sequence studies, the paired $\mathrm{m}$-sequence stimuli were presented at the same locations as the two target stimuli used for the transient study. Both m-sequence and transient target stimuli were presented for each placement of the dewar, for comparison. Three different $\mathrm{m}$ sequence stimuli were used: 1) Counter-phase stimuli. Paired target patterns were counter-phase modulated by two orthogonal short binary $\mathrm{m}$-sequences between two states (in-phase and off-phase). Subjects rested for $\sim 5-10 \mathrm{~s}$ after each of 6 binary m-sequence trials. 2) In-phase stimuli. Paired target stimuli were modulated between two different contrast levels (in-phase) of the same patterns by two orthogonal binary short m-sequences. The key feature of this stimulus is that it can be decomposed as a steady grating pattern component and a counter-phase modulated grating pattern component. 3) Unpatterned stimuli. Paired unpatterned stimuli were modulated above and below the background mean luminance by two orthogonal short binary m-sequences. MEG responses were digitized at $200 \mathrm{~Hz}$.

The nonlinear dynamics and interactions between the spatial locations were revealed by the crosscorrelations between the response and the input $\mathrm{m}$-sequences.

\section{RESULTS}

The dashed curves in Fig. 1 are evoked neuromagnetic responses to the simultaneous presentation of the two stimuli, whereas the solid curves represent the sum of the responses to the individual presentations of the two stimuli. The dashed and solid curves should be approximately equal in peak amplitude if the spatial interactions between the stimuli were linear. On the other hand, a (large) difference between the two curves suggests a (strong) nonlinear interaction. Figs. 1a and 1c show responses of 2 sensors for stimuli $0.93^{\circ}$ apart for subject WEN. Responses in Figs. $1 \mathrm{~b}$ and $1 \mathrm{~d}$ are responses to the stimulation with greater separation $\left(1.6^{\circ}\right)$. The results indicate stronger nonlinear interactions for stimuli which are closer together. Figs. le and if compare responses to paired stimuli (99\% contrast) at different eccentricities for subject CA: in Fig. 1e the stimulus eccentricity and distance were $0.8^{\circ}$ and $0.61^{\circ}$, respectively; whereas the stimulus eccentricity and distance were $3^{\circ}$ and $2.3^{\circ}$ for the responses in Fig. 1f, respectively. Figs. Ig and $1 \mathrm{~h}$ are responses to the paired stimuli ( $4.5 \mathrm{c} / \mathrm{d}, 3^{\circ}$ eccentricity) at two different
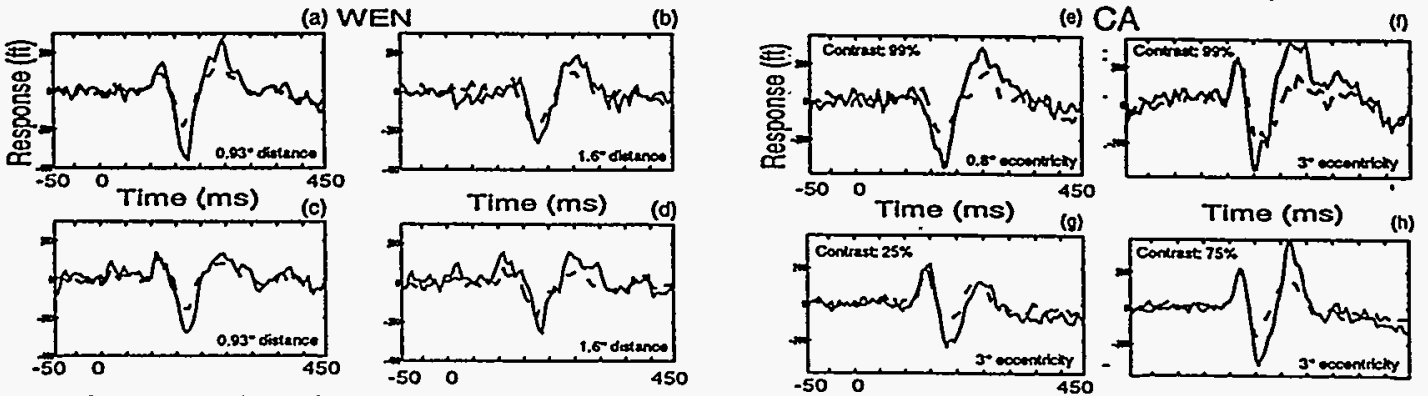

Figure 1. Spatio-temporal interactions. 1a-d: Subject WEN, the stimuli were $5.9 \mathrm{c} / \mathrm{d}, 99 \%$ contrast, and $1.6^{\circ}$ eccentricity. Ie-h: Subject $\mathrm{CA}$, the distance between the paired sitimuli is $0.61^{\circ}$ in (e), and $2.3^{\circ}$ in $(f),(g)$, and $(h)$. 
contrast levels $25 \%$ and $75 \%$, respectively for $\mathrm{CA}$.

Fig. 2 shows measured 2nd-order cross-correlations for subjects WEN and HWC using counter-phase and in-phase stimuli. The contours of these cross-correlations provide information on neural circuitry structures. The kind of contour picture in Fig. 2a suggests simple feedforward (cascade) connections, whereas the more complex pictures in Figs. $2 b$ and $2 c$ suggest involvement of more complex neural circuitry. Fig. 3 shows the power spectra (using short-time FFT, duration: $115 \mathrm{~ms}$, step: $20 \mathrm{~ms}$ ) of waveforms (subject CA) stimulated by transient stimuli $(50 \%$ contrast) at 3 different SFs $(2.0,4.5$, and $8.5 \mathrm{c} / \mathrm{d})$. The equipment and system noise $(60 \mathrm{~Hz})$ was suppressed using a threshold technique and low-pass filtering. Stronger $40 \mathrm{~Hz}$ coherent oscillations under stimulus conditions of $\mathrm{SF}=4.5 \mathrm{c} / \mathrm{d}$ (Fig. 3c) is evidenced, compared with other $\mathrm{SF}$ conditions (Figs. 3a and 3d).

(a)

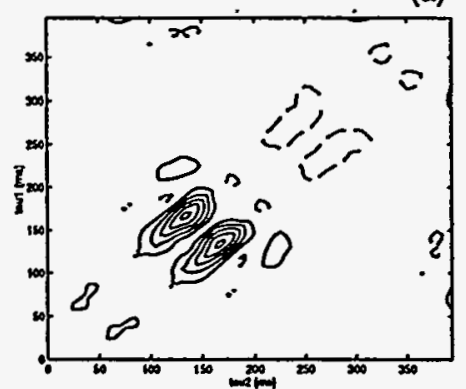

(b)
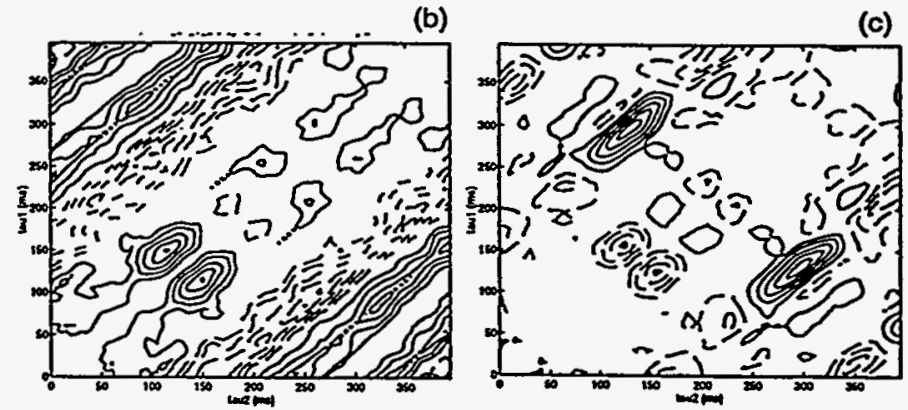

Figure 2. Measured 2nd-order cross-correlations (system kernels). Positive and negative contour values are shown as solid- and dashed-lines, respectively. (a): Subject HWC, counter-phase stimuli, $1.6^{\circ}$ eccentricity, $25 \%$ contrast, $4.5 \mathrm{c} / \mathrm{d}$. (b): Subject WEN, counter-phase stimuli, $1.6^{\circ}$ eccentricity, $25 \%$ contrast, $2.0 \mathrm{c} / \mathrm{d}$. (c): Subject HWC, in-phase stimuli, $3^{\circ}$ eccentricity, contrast change from $20 \%$ to $80 \%, 4.5 \mathrm{c} / \mathrm{d}$.
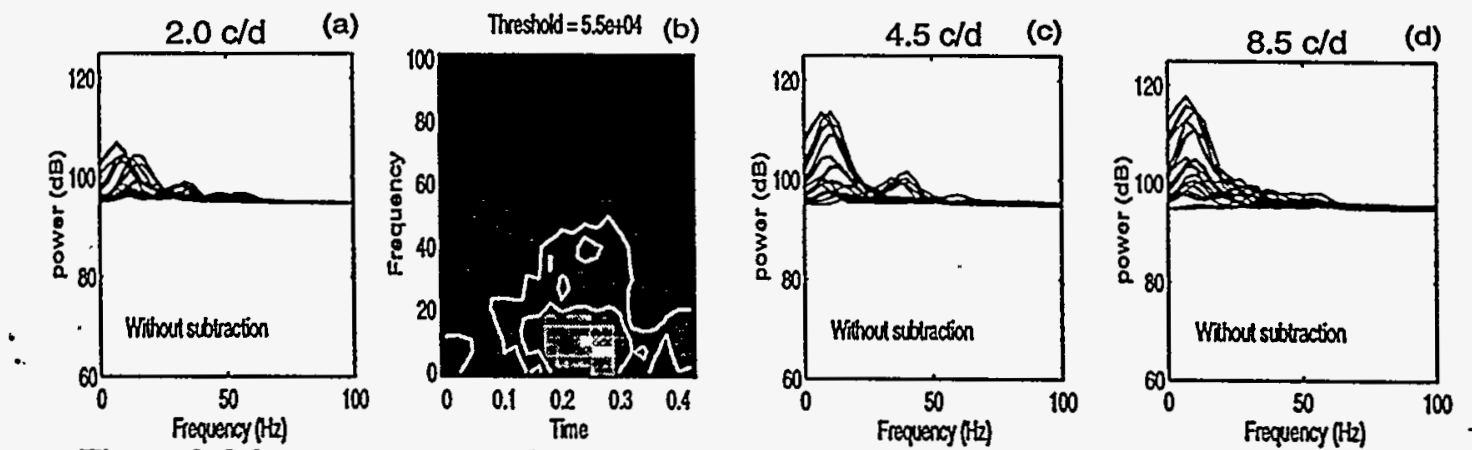

Figure 3. Measurements of coherent oscillations using short-time Fourier transform. Subject $\mathrm{CA}, 1.6^{\circ}$ eccentricity, $50 \%$ contrast. (a): $\mathrm{SF}=2.0 \mathrm{c} / \mathrm{d}$. (b) and (c): $\mathrm{SF}=4.5 \mathrm{c} / \mathrm{d}$. (d): $\mathrm{SF}=8.5 \mathrm{c} / \mathrm{d}$.

For a more quantitative analysis of nonlinear spatial summation, we introduce a summation Index (SI) by dividing the peak amplitude (e.g., the dashed curve in Fig. 1a) of the response to paired stimuli by the peak amplitude (e.g., the solid curve in Fig. 1a) of the response to the sum of the two individual stimulation conditions. An index value $\mathrm{SI}=1$ indicates a linear summation, whereas $\mathrm{SI}<1$ indicates a nonlinear suppressive interaction. For example, an SI $<0.5$ means that at least one of the peak amplitudes for single stimulus presentations was larger than that of the simultaneous stimulation. SIs were calculated for the strong second negative peak of the waveforms in Figs. 1a-d for subjects WEN and CA (not shown in Fig. 1), and are shown in Table 1.

Table1. Summation Index (SI) for two stimulus distances and their ratio

\begin{tabular}{|l|l|l|l|}
\hline Subject & SI for Distance $0.93^{\circ}$ & SI for Distance $1.6^{\circ}$ & Ratio \\
\hline CA (Sensor \#1) & 0.57 & 0.76 & 0.75 \\
\hline CA (Sensor \#6) & 0.44 & 0.58 & 0.76 \\
\hline WEN (Sensor \#2) & 0.52 & 0.71 & 0.73 \\
\hline WEN (Sensor \#7) & 0.55 & 0.72 & 0.76 \\
\hline
\end{tabular}

\section{DISCUSSION AND CONCLUSIONS}

The results in Fig. 1 indicate that the response waveforms evoked by transient stimuli showed strong nonlinear summation for shorter separations between the two stimuli. Both subjects (in Table 1) showed strong nonlinear summations for shorter distances between the two stimuli. The interactions were weaker when the distance increased (as indicated by larger SI values). As discussed above, the distance of spatial interactions may be larger for 
reciprocal connections than for feedforward connections ( $\mathrm{SF}$ channels). Therefore, it is possible that the interactions resulted from both feedforward connections and reciprocal connections when the two stimuli were closer. As the distance increases, the involvement of feedforward connections decreases, and thus the spatial interactions may be dominated by reciprocal connections. It was also found in Table 1 that the SI ratio between different separations was quite consistent between subjects. Figs. le and if indicate that the foveal responses have smaller spatial interaction areas than peripheral responses. The SI values for the 1st negative peak and the 2nd positive peak in Fig. Ig (25\% contrast, $3^{\circ}$ eccentricity) are 0.48 and 0.77 , and are 0.50 and 0.46 in Fig. $1 \mathrm{~h}$ ( $75 \%$ contrast, $3^{\circ}$ eccentricity), respectively. It appears that the interaction for the 2 nd positive peak is more sensitive to the contrast change. It is less likely that stimulus contrast would change the RF size of an SF channel, therefore, the interaction evidenced at the higher contrast level in the 2 nd positive peak may primarily result from the reciprocal connection (Singer [1] and Eckhorn et al., [2] have shown that coherence oscillations were sensitive to visual stimulus parameters such as position, orientation, movement direction and velocity, etc. Here, we show that the reciprocal connection may also be sensitive to the stimulus contrast). The SI values for the 1st positive peak in Figs. 1f, $1 \mathrm{~g}$, and $1 \mathrm{~h}$ are 1.06, 0.84, and 0.92 respectively, which indicates that the spatial summation for this peak is more linear.

From the m-sequence studies, the 1st- and 2nd-order cross-correlations were measured using unpatterned, counter-phase, and in-phase stimulation conditions. In general, strong 2 nd- (nonlinear component) but weak 1storder (linear component) cross-correlations for the unpatterned and counter-phase stimulus conditions resulted, whereas a strong 1st-order cross-correlation was apparent when in-phase stimuli were used. The estimated crosscorrelations have been further used for testing possible neural feedforward and reciprocal connections (structures) and mechanisms underlying spatial nonlinear interactions. The contours of Fig. 2a is typical for the 2nd-order unpatterned kernels and some counter-phase kernels under specific stimulus conditions. This kind of kernel picture suggests simple feedforward (cascade) connections, as corroborated by computer simulations [10]. On the other hand, the more complex pictures of the 2nd-order counter-phase and in-phase kernels (under certain stimulus conditions) in Figs. $2 b$ and $2 c$ suggest involvement of more complex neural circuitry (parallel, and/or feedback, etc.). Ongoing efforts have been directed to find appropriate neural circuitry models (e.g., the recurrent thalamic-corticalthalamic feedback connection and/or cortico-cortical reciprocal connection, etc.) which can generate these kemel pictures using computer simulations.

Fig. 3(b) and (c) indicate possible $40 \mathrm{~Hz}$ coherent oscillations under stimulus conditions of $S F=4.5 \mathrm{c} / \mathrm{d}$ and $50 \%$ contrast. The $40 \mathrm{~Hz}$ component is notably weaker under other SFs (Figs. 3a and 3d) and contrast conditions (not shown), which suggests that the coherent oscillations (and thus reciprocal connections) may be sensitive to stimulus parameters such as SFs and contrast levels. These results agree with our results from studies of spatiotemporal interactions, as discussed above.

In summary, the three methods (measurements of spatio-temporal interactions, system kernels, and coherent oscillations) used in this study may provide reliable measurements for understanding complex neural circuitry structures with reciprocal connections.

\section{REFERENCES}

[1] Singer, W. Development and plasticity of cortical processing architectures. Science, 1995, 270:758-764.

[2] Eckhom, R., Bauer, R., Jordan, W., Brosch, M., Kruse, W., Munk, M., and Reitboeck, H. Coherent oscillations: a mechanism of feature linking in the visual cortex? Biol. Cybern., 1988, 60:121-130.

[3] Li, C.Y. and Li, W. Extensive integration field beyond the classical receptive field of cat's striate cortical neurons--classification and tuning properties. Vision Res., 1994, 34:2337-2355.

[4] Polat, U. and Sagi, D. Lateral interactions between spatial channels: Suppression and facilitation revealed by lateral masking experiments. Vision Res., 1993, 33:993-999.

[5] Ts'o, D., Gilbert, CD, and Wiesel, T. Relationships between horizontal interactions and functional architecture in cat striate cortex as revealed by cross-Correlation analysis. Neurosci., 1986, 6:1160-1170.

[6] Marmarelis, P.Z. and Marmarelis, V.Z. Analysis of physiological system: the white noise approach. New York: Plenum Press, 1978.

[7] Chen, H.-W., Ishii, N., and Suzumura, N. Structural classification of non-linear systems by input and output measurements. Int J Systems Sci., 1986, 17:741-774.

[8] Chen, H.-W., Jacobson, L.D., and Gaska, J.P. Structural classification of multi-input nonlinear systems. Biological Cybernetics, 1990, 63:341-357.

[9] Chen, H.-W. Modeling and identification of parallel nonlinear systems: structural classification and parameter estimation methods. Proceedings of The IEEE, 1995, 83:39-66.

[10] Chen, H.-W., Aine, C.J., Best, E., Ranken, D., Harrison, R.R., Flynn, E.R., and Wood, C.C. Nonlinear analysis of biological systems using short m-sequences and sparse-stimulation techniques. Annals of Biomedical Engineering, 1996, 24:4 (In press).

\section{ACKNOWLEDGMENT}

This work was supported in part by a grant from NEI (EY08610) and by the United States Department of Energy (DOE) Contract W-7405-ENG-36. 